\title{
Consistent Hydrodynamic and Monte-Carlo Simulation of SiGe HBTs Based on Table Models for the Relaxation Times
}

\author{
B. NEINHÜS*, S. DECKER, P. GRAF, F. M. BUFLER and B. MEINERZHAGEN \\ Institut für Theoretische Elektrotechnik und Mikroelektronik, Universität Bremen, Kufsteiner Strasse, \\ Postfach 3304 40, 28334 Bremen, Germany
}

\begin{abstract}
Good agreement between a hydrodynamic and a Monte-Carlo device model is demonstrated in this paper for an advanced SiGe Heterojunction Bipolar Transistor. This result is based on two principles: 1) Extraction (from the Monte-Carlo bulk model under homogeneous conditions) of the relaxation times $\tau$ at discrete points of the parameter space spanned by the Ge-content $x$, doping density $N$, carrier temperature $T_{C}$ and lattice temperature $T_{L}$. 2) Modeling of the relaxation times $\tau\left(x, N, T_{C}, T_{L}\right)$ by splines.
\end{abstract}

Keywords: Silicon-Germanium (SiGe), Hetereo Bipolar Transistor (HBT), hydrodynamics, transport parameter, spline model, GHDM, Monte-Carlo

\section{INTRODUCTION}

Among all $\mathrm{SiGe}$ device concepts, SiGe Heterojunction Bipolar Transistors (HBTs) currently have the highest potential for commercial applications. In order to support the design of $\mathrm{SiGe}$ HBTs, accurate and efficient device simulation tools are necessary. However, though even some of the commercially available simulators offer the capability of simulating heterojunction devices, reliable transport parameters for these devices are not available for most design tasks. The under- lying reason for this dilemma is that the $\mathrm{Ge}$ content $x$ is variable in SiGe devices, which has added an additional dimension to the problem of determining transport parameters.

For example, the relaxation times of a hydrodynamic (HD) model for SiGe HBTs $\tau\left(N, x, T_{C}\right.$, $T_{L}$ ) depend on four independent quantities instead of three, namely the total doping density $N$, the Ge content $x$, the carrier temperature $T_{C}$ and the lattice temperature $T_{L}$. Therefore the traditional approach of extracting transport parameters predominantly from experimental data, which worked

\footnotetext{
* Corresponding author.
} 
well for silicon for a long time, is no longer feasible for $\mathrm{SiGe}$ devices because reliable experimental data, especially for strained $\mathrm{SiGe}$, are hardly available.

\section{TRANSPORT PARAMETERS}

In order to overcome this problem the comprehensive and experimentally verified Monte-Carlo bulk transport model described in [1] was applied to generate transport parameters for the driftdiffussion (DD) or HD device simulations at discrete mesh points of the 4D space spanned by $N, x, T_{C}, T_{L}$.

To generate the smooth functions $\tau\left(N, x, T_{C}\right.$, $T_{L}$ ), that are needed for example for HD device simulations from the resulting table of transport data, a flexible monotonicity preserving spline interpolation scheme has been developed.

\section{THE SPLINE MODEL}

Compared to the traditional approach of using closed form analytic expressions with only few model parameters for $\tau\left(N, x, T_{C}, T_{L}\right)$ our spline approximation scheme has several advantages: For example, it adapts itself automatically to model extensions like an extended range of $N, x$, $T_{C}$ or $T_{L}$. Moreover, it is easy to control the accuracy of the spline interpolation by just generating a denser table of transport data for the range of $N, x, T_{L}, T_{C}$, that is of highest interest. In addition, our method is also capable of working on a non-rectangular grid in the $N-x-T_{L^{-}}$ $T_{C}$ space.

The underlying algorithm considers the functional dependencies of the transport parameters in two stages. First, the dependence on variables not being influenced by device simulation like $N, x_{G e}$ and sometimes $T_{L}$ are considered by multilinear interpolation. In order to process data on a nonrectangular grid the algorithm performs a multidimensional search of the nearest neighbour data points suitable for the interpolation.
The second stage deals with dependencies on variables of the hydrodynamic equations itself, like the carrier temperature $T_{C}$. This is considered by using a variant of cubic splines called Aкima Subsplines [2]. The spline curves generated by this method do not contain artificial oscillations which can cause artificial modeling results or convergence problems for the solution algorithm.

The final result that influences the memory requirements of the device simulator is a set of spline coefficients for each grid point of the device. For medium grid sizes (3000 points) roughly 20 MBytes of memory are necessary to hold the spline coefficients.

\section{SIMULATION RESULTS}

In order to verify the validity of our modeling approach we have simulated the two-dimensional $\mathrm{SiGe}$ HBT structure shown in Figure 1. The structure is very demanding for numerical device models because of its narrow base and piecewise constant profiles for $N$ and $x$ that give rise to abrupt junctions and steps in the valence and conduction band edges. Consequently this device is well suited for testing the modeling accuracy of

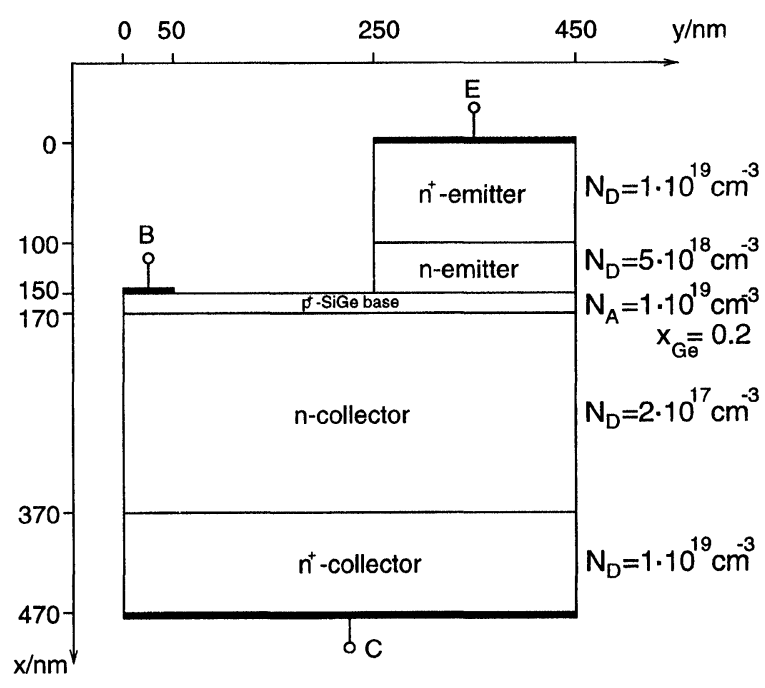

FIGURE 1 The 2D SiGe-HBT test structure. 
classical device simulation in comparison to Monte-Carlo device simulation. The structure has been simulated by three different models. The first model is the DD model. The second model is an extension of the Generalized HD Model reported in [3] for devices with positiondependent band structure. Moreover, the heat flux reduction that has been proven to be beneficial for ultra short MOSFETs [4] has been adopted. The third model is a newly developed Monte-Carlo device model for SiGe heterojunction devices. Details of this MC model will be published elsewhere. Since all transport parameters of the DD and HD simulators have been derived from the SiGe MC bulk model and because the MC device model used exactly the same band structure and scattering models as the MC bulk simulator, all three models are fully consistent under homogeneous material and field conditions. Moreover, all three device models use exactly the same offsets for the valence and conduction band edges.

The results of the $2 \mathrm{D}$ simulations for $V_{\mathrm{BE}}=0.75$ $\mathrm{V}, V_{\mathrm{CE}}=1 \mathrm{~V}$ and $300 \mathrm{~K}$ are summarized in Figures 2-5 for the electric potential, the dynamic temperature, the drift velocity and the electron density. In all cases the results of the three models are shown along the vertical line at $y=450 \mathrm{~nm}$. It can be seen that at the base collector junction even in the MC model the drift velocity is more than a factor of two higher than the maximum drift velocity under homogeneous field conditions. Despite this overshoot, which would be extremely large for Si-based devices, it can be clearly stated that the hydrodynamic results are in good agreement with the MC-results. Especially the electron density profile in the base and the space charge region, which is important for the transient behavior of the HBT, agrees well for the HD

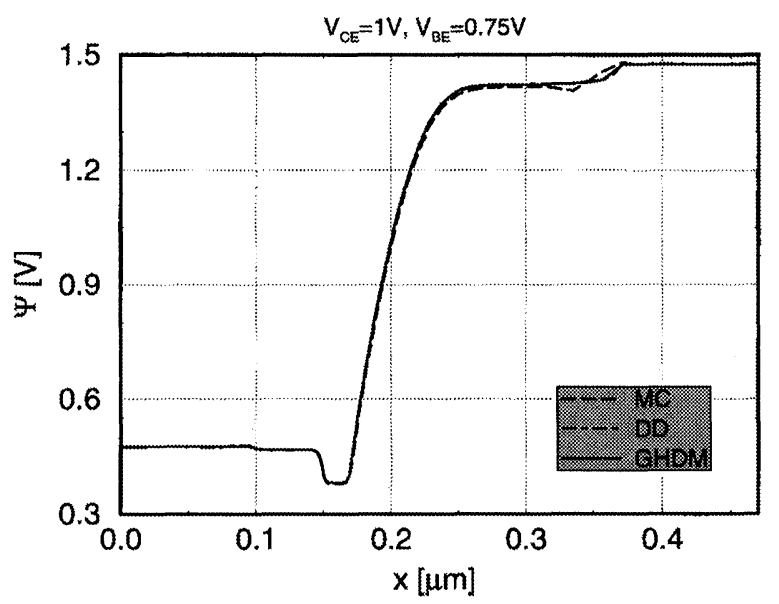

FIGURE 2 Comparison of the electrostatic potential profiles resulting from the DD, HD and MC models. All models are in good agreement.

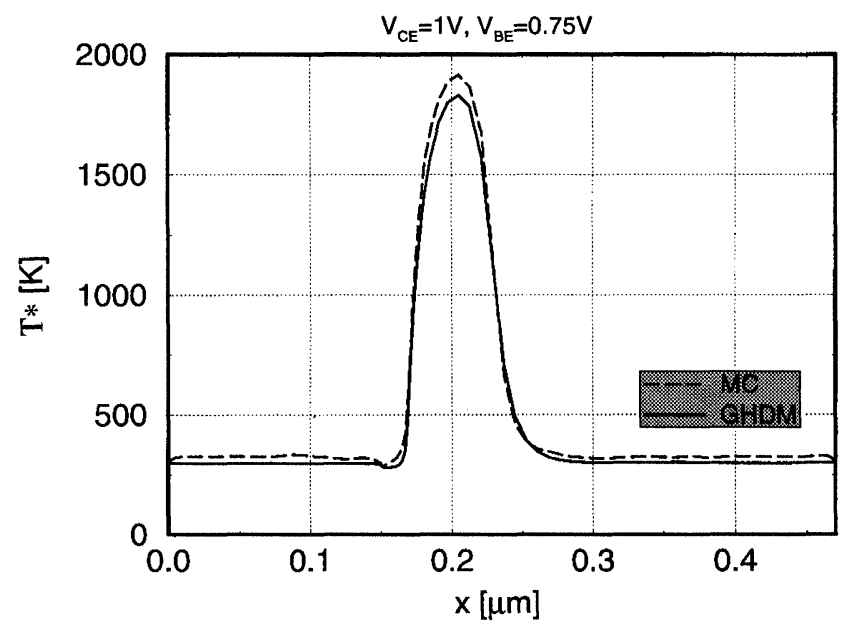

FIGURE 3 Comparison of the dynamic temperature profiles resulting from the HD and MC models. Both model are in good agreement. 


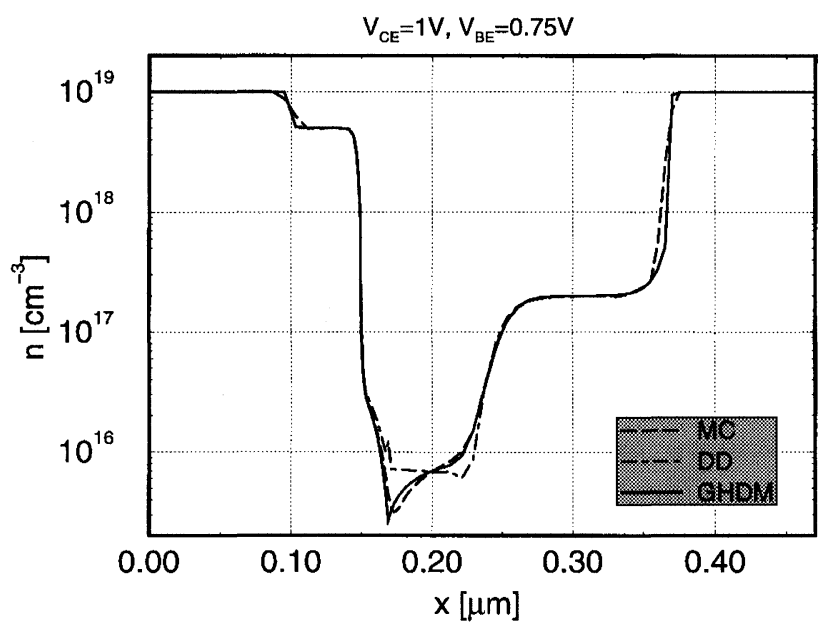

FIGURE 4 Comparison of the electron densities resulting from the DD, HD and MC models. The HD and MC density distributions are in good agreement. The DD model deviates substantially.

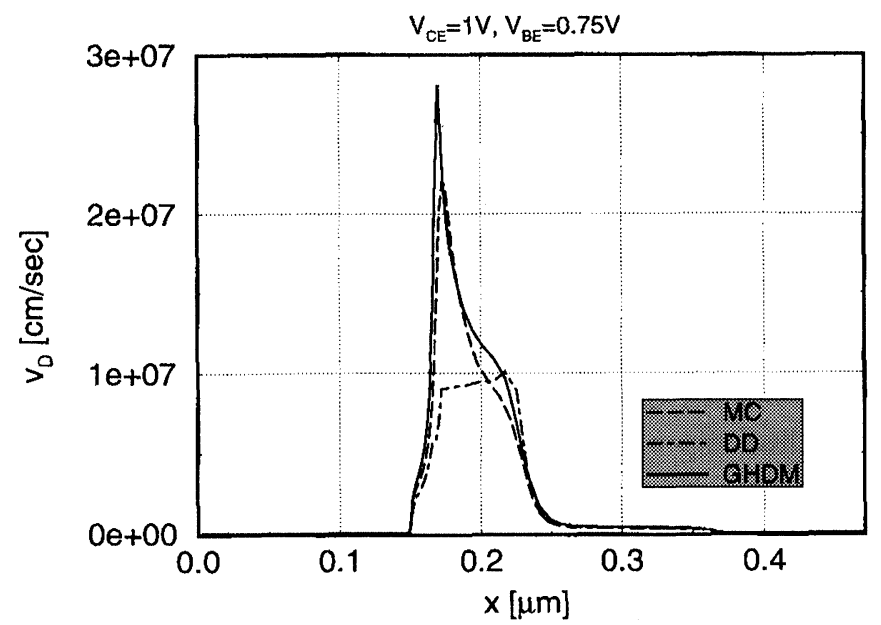

FIGURE 5 Comparison of the drift velocity profiles resulting from the DD, HD and MC models. Please note the velocity overshoot which is extreme for Si-based devices.

and the MC simulation. On the other hand, the DD simulation deviates much more from the MCreference, which may lead to intolerable errors of the DD-model for agressively scaled SiGe HBTs in the near future [5].

To the best of our knowledge the results reported in this paper represent the first 2D simulations of a SiGe HBT with fully consistent $\mathrm{DD}, \mathrm{HD}$ and $\mathrm{MC}$ device models.

\section{Acknowledgements}

This work was supported in part by the Bundesministerium für Bildung, Wissenschaft, Forschung und Technologie under Contract No. 01M2416A and by Siemens AG in Munich. The authors like to thank H. Förster, D. Nuernbergk and F. Schwierz from the Technical University of Ilmenau for proposing the SiGe HBT in Figure 1 and several fruitful discussions. 


\section{References}

[1] Bufler, F. M., Graf, P., Meinerzhagen, B., Adeline, B., Rieger, M. M., Kibbel, H. and Fischer, G. (1997). "Analysis of electron transport properties in unstrained and strained $\mathrm{Si}_{1-x} \mathrm{Ge}_{x}$ alloys", IEEE Trans. Semicond. Technol. Modeling and Simulation, 1.

[2] Müllges, G. E. and Reutter, F. (1993). Numerik-Algorithmen mit ANSI-C Programmen, BI-Wissenschaftsverlag, Mannheim, 2nd edition.

[3] Thoma, R., Emunds, A., Meinerzhagen, B., Peifer, H. J. and Engl, W. L. (1991). "Hydrodynamic equations for semiconductors with nonparabolic bandstructures", IEEE Trans. Electron Devices, 38, 1343-1352.

[4] Bork, I., Jungemann, C., Meinerzhagen, B. and Engl, W. L. (1994). "Influence of heat flux on the accuarcy of hydrodynamic models for ultrashort $\mathrm{Si}$ MOSFETs", in NUPAD Tech. Dig., Honolulu, 5.

[5] To be published at ESSDERC 97.

\section{Authors' Biographies}

Burkhard Neinhüs received the Diploma in physics from the RWTH Aachen, Germany, in 1995. In 1996 he joined the Insitut für Theoretische Elektrotechnik und Mikroelektronik at the University of Bremen and is working on hydrodynamic device simulations.

Stefan Decker received the Diploma in electrical engineering from the RWTH Aachen, Germany, in 1993. From 1993 to 1995 he worked at the Institut für Theoretische Elektrotechnik, RWTH Aachen, on hydrodynamic and Monte-Carlo simulation methods. Since 1995 he was working at the Insitut für Theoretische Elektrotechnik und Mikroelektronik at the University of Bremen.

Peter Graf received the Diploma in physics from the RWTH Aachen (Technical University of Aachen), Germany, in 1991. At the Institut für Theoretische Elektrotechnik, RWTH Aachen, he then worked on the Monte-Carlo simulation of homogeneous systems and its benefits for hydrodynamic charge transport parameters. After joining the Institut für Theoretische Elektrotechnik und Mikroelektronik, Universität Bremen, he dealt with algorithms for Monte-Carlo device simulation and their implementation into the institute's Monte-Carlo heterojunction device simulator.

Fabian M. Bufler studied physics at the TU Braunschweig and RWTH Aachen (both Germany) including an academic year at the Université de Grenoble I (France) with a scholarship of the Studienstiftung des deutschen Volkes and received the Dipl.-Phys. degree in 1992. Since then he was working as a research assistant at the Institut für Theoretische Elektrotechnik, RWTH Aachen, and moved in 1995 together with the group of Prof. Bernd Meinerzhagen to the Institut für Theoretische Elektrotechnik und Mikroelektronik, Universität Bremen (Germany). His main research interests are concerned with transport theory and full band Monte Carlo simulation for SiGe based devices.

Bernd Meinerzhagen received the Dipl.-Ing. degree in electrical engineering in 1977, the Dipl.Math. degree in mathematics in 1981, the Dr.-Ing. degree in electrical engineering in 1985 and the "venia legendi" in 1995 all from the RWTH Aachen (Germany). From 1978 to 1986 as a Research and Teaching Assistant at the RWTH Aachen he worked mainly on the development of numerical device modeling codes. In 1986 he joined AT\&T Bell Laboratories in Allentown, PA, as a Member of Technical Staff, where he developed advanced numerical models for MOS substrate and gate currents. He went back to the RWTH Aachen in 1987 and became head of the research and development group for Silicon technology modeling and simulation (TCAD). In 1995 he was appointed Professor at the University of Bremen, where his current research interests include TCAD and the theory of electromagnetic fields and networks. 

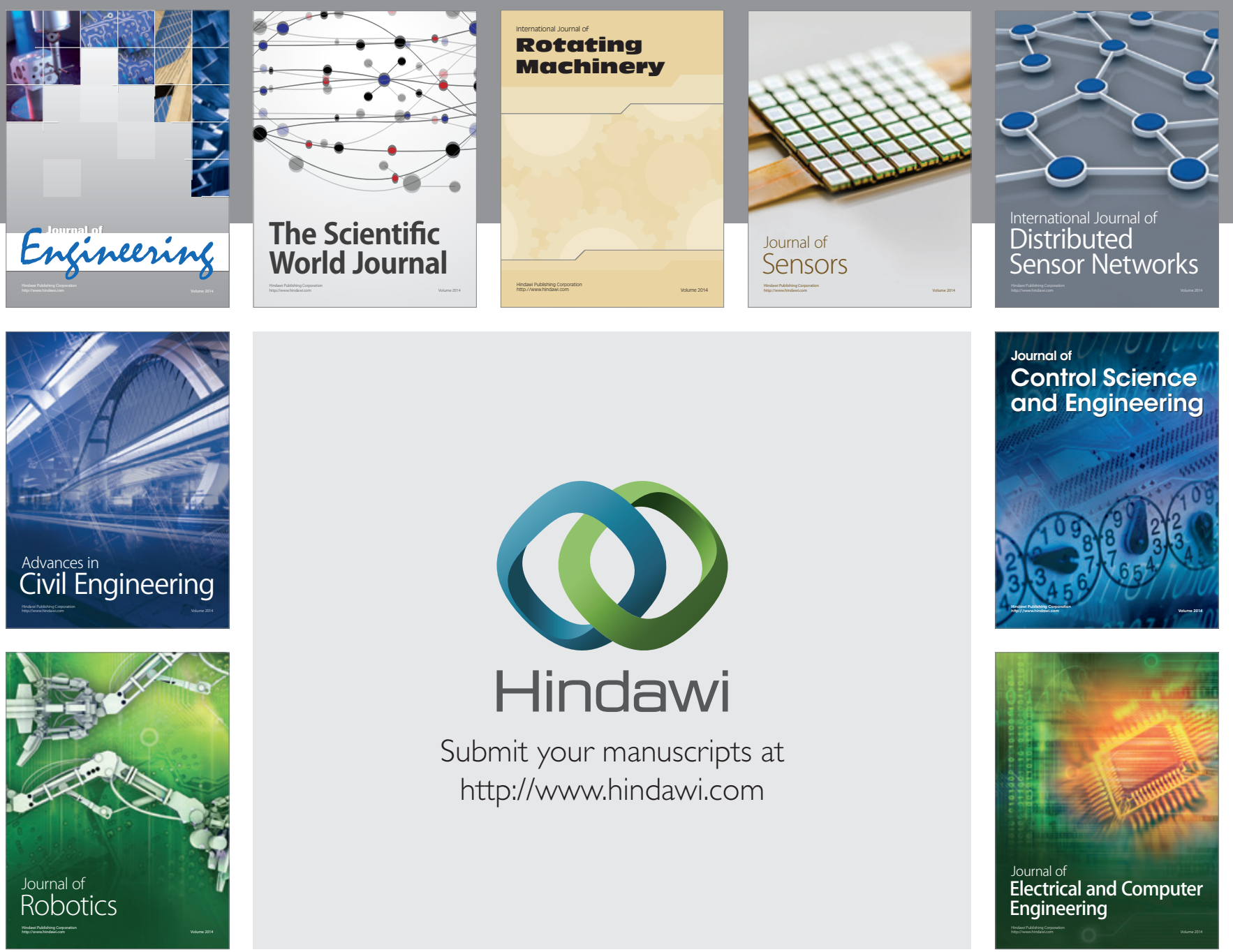

Submit your manuscripts at

http://www.hindawi.com
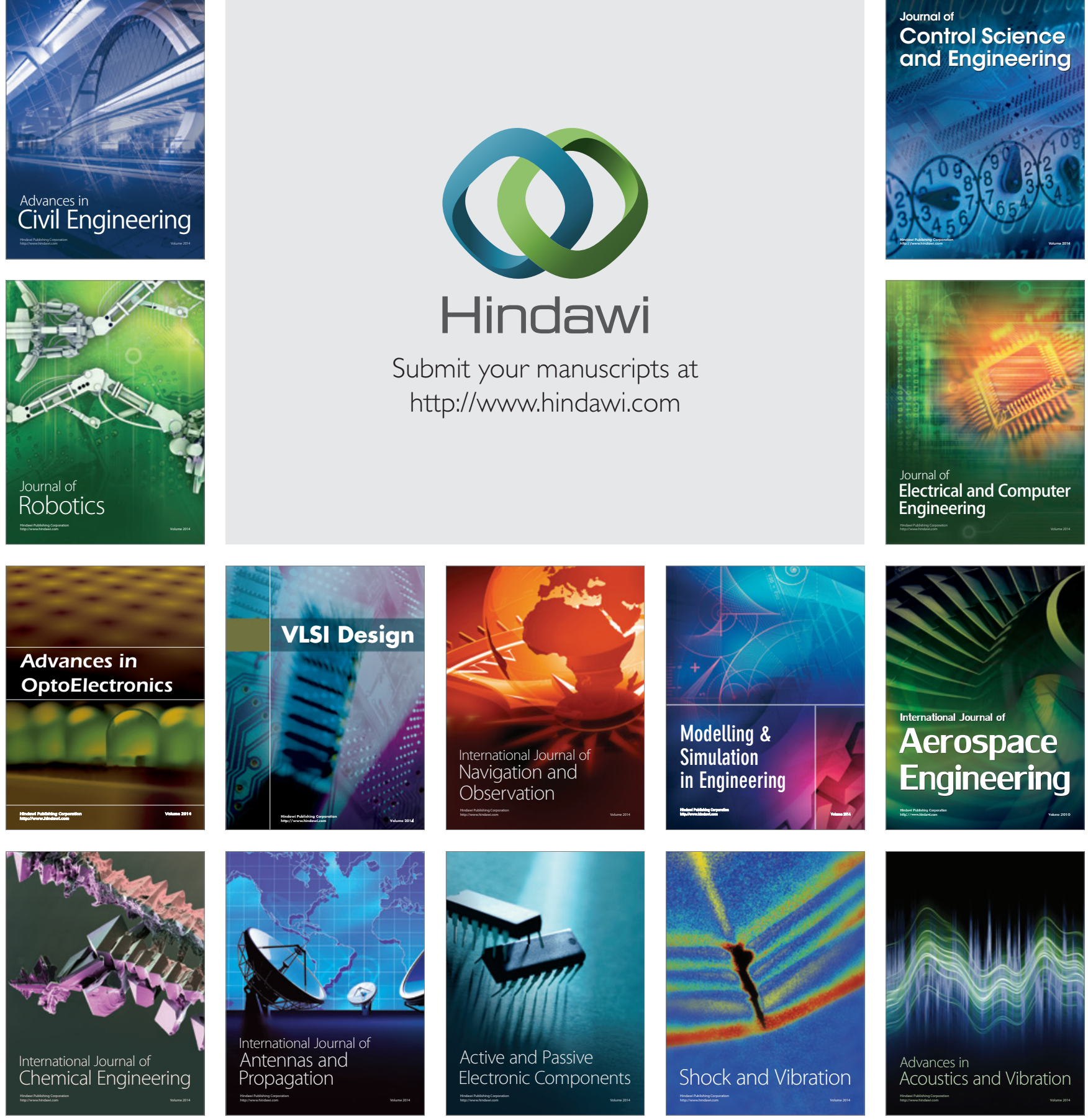